\title{
Estados Unidos en el pensamiento conservador mexicano a finales del siglo $\mathrm{XIX}^{\star}$
}

\section{Marcel Nagy}

La guerra con los Estados Unidos dejó claro para todos los actores de la política mexicana que había que contar con la presencia del coloso del norte; y tanto los conservadores como los liberales tuvieron que expresarse respecto al problema de "salvación de la nacionalidad". La respuesta de los conservadores ya estaba codificada en las décadas anteriores: la acentuación del origen hispánico y la admiración de la Europa latina (España y Francia, principalmente) sumada a la agresión de los Estados Unidos dio como fruto el anti-yanquismo, que algunas veces iba acompañado de la admisión de los resultados logrados en el norte.

Los liberales, por su parte, se decidieron por un mimetismo: para proteger al país de los desastres posteriores, y subrayaron la importancia de llegar a un desarrollo semejante al visto en los Estados Unidos. ${ }^{1}$

Estados Unidos sacudió "la conciencia de los mexicanos"2, que empezaron a cristalizar su ideología. Para triunfar en las elecciones los monarquistas y otros grupos decidieron organizar el partido conservador. Ellos fueron los primeros en 1849. La base de su pensamiento era la visión de una sociedad realizada según los valores religiosos y fundamentada en el pasado que servía como "fuente de inspiración para planear el futuro" e iba acompañado por un fuerte sentimiento antiyanqui. ${ }^{3}$

Para lograr ese anhelo común de "salvar la nacionalidad mexicana", solución en el extranjero: los conservadores en Europa (Maximiliano) y los liberales en los Estados Unidos. Estos últimos, en espera de préstamos, en 1859 firmaron el tratado McLane-Ocampo ${ }^{5}$, que autorizaba el tránsito libre a los "Estados Unidos y sus conciudadanos y bienes" por el istmo de Tehuantepec. ${ }^{6}$

Después del imperio de Maximiliano (1867), los liberales intentaron normalizar las relaciones con EEUU. La época de la república restaurada fueron los años de acercamiento ${ }^{7}$, pero al llegar a la presidencia, Díaz tuvo que enfrentar algunos conflictos con el vecino. Después del Plan de Tuxtepec (1876) Lerdo e Iglesias se exiliaron en los Estados Unidos y dificultarían el reconocimiento por parte de los estadounidenses. Un año más tarde, el presidente electo de EEUU, Rutheford B. Hayes, para distraer la atención de la población de su país (por el fraude electoral cometido) reavivó el tema de las discusiones fronterizas en Texas y varios diarios publicaron artículos sobre la situación en México, concluyendo que el país era incapaz de estabilizar la situación política y de tal manera era menester el establecimiento de un protectorado. Tales declaraciones provocaron indignación en el país, y Díaz pudo "presentarse como defensor de la resistencia a la agresividad del país del Norte" consiguiente fortalecer su posición."

Ya los primeros años de la gestión de Díaz generaron conflictos entre los dos países, y a pesar de que en las siguientes décadas la economía mexicana estará influenciada por la presencia del capital norteamericano, don Porfirio intentó mantener un equilibrio entre los Estados Unidos y Europa respecto a la influencia extranjera. Fruto de esta política fue la llamada doctrina Díaz, que repensaba la

\footnotetext{
${ }^{*}$ La ponencia está basada en investigaciones realizadas gracias a la beca de seis meses otorgada por la Secretaría de Relaciones Exteriores de México.

${ }^{1}$ MATESANZ IBÁÑEZ, José Antonio: "Notas sobre el conservadurismo de Francisco de Paula de Arrangoiz", in: Estudios de historia moderna de México, 1977, vol. VII (51-68): 67

${ }^{2}$ VÁZQUEZ, Josefina Zoraida: “Liberales y conservadores: diferencias y similitudes” in: Cuadernos Americanos 1997/66, (153-175): 170

3 idem

${ }^{4}$ MATEZANS, op.cit.: 67

${ }^{5}$ VÁZQUEZ, op.cit.: 82

6 “Tratado McLane-Ocampo", in: MATUTE, Alvaro: México en el siglo XIX. Fuentes e interpretaciones históricas, México, UNAM, 1992 (489-494): 489

${ }^{7}$ VÁZQUEZ, op. cit.: 91

${ }^{8}$ VÁZQUEZ, op. cit.: 99

${ }^{9}$ VÁZQUEZ, op. cit.: $97-99$
} 
doctrina Monroe, expresando discordia con el papel de "policía panamericano" de los Estados Unidos y subrayaba la importancia de la igualdad entre las naciones americanas. ${ }^{10}$ Paralelamente el gobierno de Díaz ofreció concesiones privilegiadas a grupos de inversores europeos, acompañadas con restricciones antinorteamerica-nas. ${ }^{11}$

En el pensamiento conservador y más concretamente en el monarquismo de la década de los 1840, la instalación de una monarquía en México cumplía con el deber de defender al país contra la amenaza del norte. Ya Haro y Tamariz en 1846, en una carta dirigida a sus "conciudadanos" sobre la monarquía constitucional, advertía que después de conseguir Texas, Estados Unidos no pararía e intentaría conseguir también los estados limítrofes. En su visión, la monarquía podría ser un arma contra los EEUU y en defensa de la "raza hispano-mexicana". ${ }^{2}$ Según José María Gutiérrez Estrada (monarquista de la década de los 1840) lo que estaba en peligro era la supervivencia de México en general como nación: por dentro era la desintegración del país en grupos políticos, y por fuera un contexto internacional hostil a su independencia. ${ }^{13}$ (Estas observaciones eran un tanto proféticas: se acercaba la guerra contra los EEUU): "iquizá no pasarán veinte años sin que veamos tremolar la bandera norteamericana en nuestro Palacio Nacional!"’14, escribía.

El monarquismo mexicano ya desde la década de 1840 consideraba necesario algún tipo de apoyo foráneo, sea este un príncipe o una intervención extranjera para la realización de su programa político, siempre que estos sean europeos ${ }^{15}$. Este programa fue defendido por la gran mayoría del partido conservador y varios representantes del liberalismo. La guerra contra los Estados Unidos, la constitución de 1857 y la Guerra de Tres Años radicalizaron las posturas de los diferentes grupos políticos y estos tres acontecimientos pueden considerarse como preludio del Segundo Imperio. Los liberales no pudieron reorganizar el país mediante la constitución (1857) que finalmente se convirtió en una nueva fuente de conflictos. El descontento interior y el hecho de que se formara la triple alianza de Inglaterra, España y Francia en 1861 para intervenir en México funcionaron como un motor para los monárquicos conservadores: consideraron que llegó el momento adecuado para realizar su idea de invitar a un monarca al trono del país. Por otra parte el miedo de que los Estados Unidos interviniera en México o el miedo de la anexión hizo que muchos liberales decidieran apoyar las ideas monárquicas.

En los años posteriores reaparece entre los argumentos monárquicos el de que el imperio funcionaría también como una defensa contra los EEUU. Este argumento, como era de esperar iba acompañado de una crítica frente a los liberales que desde el punto de vista conservador llevaban una política pro-yanqui, y que al fin y al cabo no querían otra cosa que un "protectorado directo de los Estado Unidos", lo que habría significado el fin de la independencia de México. ${ }^{16}$

Otro argumento muchas veces repetido a favor de la necesidad de la intervención y el imperio fue el peligro del norte, la "absorcion por los Estados Unidos". ${ }^{17}$ Aguilar y Marocho también expresó ideas semejantes: en 1866 escribía que si por alguna razón Maximiliano tuviera que abandonar el

\footnotetext{
${ }^{10}$ RIGUZZI, Paolo: “México, Estados Unidos y Gran Bretaña, 1867-1910: una difícil relación triangular”, in: Historia Mexicana, 1992, N. 163 (365-436): 413

${ }^{11}$ RIGUZZI, op. cit.: 417

${ }^{12}$ HARO Y TAMARIZ, Antonio de: Esposicion que Antonio de Haro y Tamariz dirige á sus conciudadanos, y opiniones del autor sobre la monarquía constitucional, México, Imprenta en el Arquillo de la Alcaicena, 1846: 2,5

${ }^{13}$ PALTI, Elías José: La política del disenso, México, Fondo de Cultura Económica, 1998: 11-15

${ }^{14}$ Gutiérrez Estrada, citado por: PALTI, op. cit: 15

${ }^{15}$ O'GORMAN, Edmundo: La supervivencia política novo-hispana, México, Universidad Iberoamericana, 1986: 29 y ss.

16 "Dictamen acerca de la forma de gobierno que, para constituirse definitivamente, conviene adoptar en México; presentado por la Comisión especial que en la sesión del 8 de Julio de 1863, fue nombrada por la Asamblea de Notables reunida en el cumplimiento del decreto de 16 de Junio último", in: AGUILAR Y MAROCHO, Ignacio: La familia enferma, México, JUS, 1969 (163-196): 179

${ }^{17}$ ANÓNIMO: Los traidores juzgados á la luz de la razón por la Revista Universal, México, Tipografía Mexicana, 1869: 52
} 
trono lo que esperaría a México sería la anexión a los Estados Unidos o el "restablecimiento de la República bajo la férula de los Juarez..." y otros. ${ }^{18}$ Cuando Francia decidió alejar sus tropas de México, el mismo Marocho saludó el hecho, puesto que según su visión la presencia de tropas extranjeras en el país desacredita el régimen y además fortalecería el sentimiento nacional de México $^{19}$ : la retirada de los franceses significaría que su legitimidad no fue "importada".

En 1871 la Sociedad Católica publicó un artículo bajo el título: Destino Manifiesto. ${ }^{20}$ En éste analizan el sentido y contenido de tal expresión que para el autor no significa otra cosa que "conquista", conquista de México, como lo han hecho ya con Texas y posteriormente, en 1847. Pero, no pararán en México, sigue, sino que tienen la mira en todo el mundo y cuentan con la ayuda de Rusia: "S.Petersburgo y Washington pueden sin rubor y sin traba proclamar el 'destino manifiesto' del mundo, y dándose la mano por el estrecho de Bering, soltarlo como perro rabioso contra la independencia de las naciones" ${ }^{21}$.

El indudable hispanismo conservador (basado en tradición de nostalgia hacia el sistema colonial y el rotundo rechazo de la doctrina Monroe) alrededor del cambio de siglo tuvo varias oportunidades para enunciarse. El primer Congreso Panamericano, la lucha de independencia de Cuba desde 1895, más tarde el segundo Congreso Panamericano y el primer Hispanoamericano por un lado, y la política cada vez más concreta de los Estados Unidos en las Antillas y América Central por el otro, hicieron reaccionar a los periodistas y redactores católicos de El Tiempo. Examinaremos varios artículos de 1890, 1898, 1900 y 1903.

Cuando se reunió el primer Congreso Panamericano los católicos mexicanos valoraron este encuentro en una forma nada sorprendente: el congreso sirvió a los intereses norteamericanos, cuya meta final es la de dominar todo el continente americano; todo lo que se hace a favor de la unificación de las Américas finalmente se utilizará para los fines de dominio de los Estados Unidos del Norte. Todas las iniciativas de esta índole (tratados sobre cooperación de "los bancos internacionales, ferrocarriles internacionales, navegación internacional, aduanas internacionales, tratados literarios y comerciales internacionales, lengua internacional, etc., etc.") son parte de un plan, “...de una ambición que supera á la de los más célebres conquistadores que han dominado los continentes enteros de Africa ó Asia ó Europa...", pero no mediante los viejos métodos ("invasión”), sino “...por el método nuevo moderno que consiste en matar todo tráfico de los continentes del mundo antiguo con el mundo moderno y ser ella la única fabricante de mercancías y sus dominados (todos los pueblos iberoamericanos) los consumidores sumisos y resignados de sus productos." Es necesario, agregan, que se hable de todo esto para que las nuevas generaciones sepan cuál es el futuro de Latinoamérica. ${ }^{22} \mathrm{Y}$ para subrayar cuál es la verdadera cara de la política de EEUU enumeran los ejemplos de Centroamérica, o el caso de las Islas Galápagos: "Mac Kinley ha hecho [evidentes esfuerzos] para adquirir pacíficamente del Ecuador una estación naval en el Archipiélago" ${ }^{23}$ A estos añadieron los redactores de El Tiempo, en 1903, el caso de Colombia y el estrecho de Panamá, "donde inventaron una parodia de Nación"24. Este hecho, escribían, ha causado un malestar general en América, que está dividida, y por tanto no tiene ni la unión ni la fuerza necesaria para "pelear con el coloso". Según El Tiempo, una solución podría ser el fortalecimiento de la Unión Ibero-Americana que había iniciado su labor algunos años antes y en la cual participan también las delegaciones del Portugal y España. ${ }^{25}$ En este

\footnotetext{
${ }^{18}$ AGUILAR Y MAROCHO, Ignacio: Reflexiones sobre la carta escrita por el Excmo. Sr. D. Francisco de Arrangoiz y Berzabal á S. M. El Emperador de Méjico, Madrid, Imprenta á cargo de Francisco Roig, 1866: 15

${ }^{19}$ Carta de Ignacio Aguilar y Marocho a Maximiliano, 12 de noviembre de 1866, in: CONDUMEX, IX-I, carp. 8 , doc. 915 .

${ }^{20}$ ANÓNIMO: Destino Manifiesto. Es un folleto de 4 páginas incluidas en un tomo más voluminoso. Lamentablemente solo encontramos estas páginas. Tal vez fue un artículo publicado en El Pensamiento Católico. Es seguro que fue imprimido en Colima en 1871 por la Sociedad Católica.

${ }^{21}$ ANÓNIMO: Destino...: 296

22 "El Congreso Pan-Americano", in: El Tiempo 27 de diciembre de 1890

23 "El peligro del Norte", in: El Tiempo, 6 de junio de 1900

24 "El año de 1903", in. El Tiempo, 31 de diciembre de 1903

25 "Dos congresos internacionales", in: El Tiempo, 12 de julio de 1900
} 
asunto los redactores de El Tiempo y don Porfirio coincidían. En el informe que dirigió Díaz al congreso el 16 de septiembre de 1900 afirmó sobre el tema de la conferencia de la Unión y la invitación que había recibido: "Desde luego hemos manifestado nuestra buena disposición para concurrir a ese Congreso, cuyas tendencias, apoyadas en la comunidad de lengua y en el origen de nuestra actual civilización, son para nosotros muy simpáticas". ${ }^{26}$

El caso de la revolución cubana (1895-98) y la posterior guerra entre los Estados Unidos del Norte y España, por su complejidad -desde el punto de vista del pensamiento católico-conservador- condujo a observaciones interesantes. El "enemigo de la raza", los Estados Unidos, naturalmente es visto por los católicos como "el malo", que conquista a Cuba, que al mismo tiempo se rebeló contra España. En conceptos católico-conservadores, España era la Patria, con mayúscula, que durante todo el siglo XIX sufrió, desde Trafalgar hasta Cuba y sus "hijos ingratos conspiran contra ella" y, como si eso no fuera bastante, las contrariedades de sus políticos la hacen más pobre y débil. Frente a esta "Nación Heroica", el enemigo ("coloso") es rico, con ambición y orgullo. España está sola y necesita ayuda, pero las naciones se alían con los yanquis. ${ }^{27} \mathrm{El}$ gobierno mexicano tiene que ser neutral en esta guerra, pero el pueblo debe ser partidario de España, “...no sólo porque ella es justa, sino porque los que la combaten son los enemigos de nuestra raza, de nuestras tradiciones y de nuestra autonomía, los que nos robaron la mitad de nuestro territorio y lyncharon a nuestros compatriotas en Texas, Nuevo México y Colorado"28. América y principalmente América Latina traicionó a España, concluye el artículo anterior: "¡Fuiste madre de América y ella te niega!..." ${ }^{29}$ En 1900 escribe el articulista de El Tiempo que mucho cubanos ya se han dado cuenta de que fue un error aliarse con los yanquis sólo por odiar a España, que Cuba se transformó ahora en un esclavo de los Estados Unidos, pero esa fue una desilusión muy merecida y además la nueva situación político-administrativa de Cuba no se diferencia mucho de la del régimen derrocado por la revolución. El poder que gobierna en la isla, además, es el más absoluto que jamás ha tenido Cuba. ${ }^{30}$

La figura de José Martí, a pesar de su "anti-yanquismo", no pudo ser valorada de una forma positiva en el pensamiento conservador. Como se rebeló contra España para los redactores de La Voz de México no fue más que un "poeta conspirador", el "iluso Martí titulado presidente delegado de la imaginaria República Cubana" y "corifeo del trasnochado separatismo". La corriente independentista, o sea el separatismo, también fue visto como "una cosa tan descabellada".

Consumada la guerra entre EEUU y España se negoció el tratado de paz que según los editores de El Tiempo es una vergüenza para el ejército y para la patria (España), puesto que significa "la pérdida del imperio colonial". ${ }^{32}$

Esta crisis finisecular, simbolizada por la "tragedia" de Cuba, que tanto influyó en el pensamiento latinoamericano ${ }^{33}$, introdujo nuevas voces en la terminología del ensayo. Aparece "el coloso", "Calibán", la "nordomanía", los pueblos "deslatinizados", el "peligro del norte", etc., todas expresiones que subrayan las diferencias esenciales que existían entre la América hispana, o latina y la sajona, y el peligro que significaba para las naciones de la América latina el creciente imperialismo de EEUU. Muchos pensadores llamaron la atención sobre este peligro, demandando una solidaridad hispanoamericana (latinoamericana o iberoamericana). No es el "anti-yanquismo" una característica exclusiva del pensamiento conservador o católico, pero el hispanismo (por lo menos en México) sín ${ }^{34}, \mathrm{y}$ monopolizarlo sirvió como una forma de atacar a los liberales que fueron tantas veces tachados de ser "pro-yanquis" por los conservadores, para quienes los EEUU eran el liberalismo en sí.

\footnotetext{
${ }^{26}$ Un siglo de relaciones internacionales de México (A través de los mensajes precidenciales), México, Secretaría de Relaciones Exteriores, 1935: 208

27 "Nación Heroica", in: El Tiempo, 6 de julio de 1898

28 "Cuestión de yankismo", in: El Tiempo, 6 de julio de 1898

29 "Nación Heroica", in: El Tiempo, 6 de julio de 1898

30 “Cuba y los Estados Unidos", in: El Tiempo, 20 de junio de 1900

${ }^{31}$ La Voz de México, 4 de junio de 1895, citado por DUMAS, Claude: "El discurso de la oposición en la prensa clerical de México en la época de Porfirio Díaz”, in: Historia Mexicana, 1989, N 153 (243-256): 254-255

32 "Comentario del día", in: El Tiempo, 18 de agosto de 1898

${ }^{33}$ Basta hacer recordar a las obras de José Martí, José Enrique Rodó, Rubén Darío y toda la siguiente generación de ensayistas en todo el continente.

${ }^{34}$ Por ejemplo en: "Dos congresos internacionales", in: El Tiempo, 12 de julio de 1900
} 
La derrota de España tiene que ser una señal, subrayaban los editores de El Tiempo, de que los países de Hispano América están amenazados por el imperialismo norteño, y para dar mayor autenticidad a sus palabras citan a un periodista del New York Evening Journal, que se expresó de esta forma: "Para Estados Unidos de América necesitamos 'toda' Norte América, 'toda la América del Sur"”. ${ }^{35}$ Todos los planes y proyectos ${ }^{36}$ de EEUU están pensados para estos fines. Por eso se necesita la unión de "...toda la familia hispana ... [y] estrechar entre sí los vínculos de toda especie"37 y acentuar las diferencias que existen entre las dos partes de América: "Porque hacia el Norte nos dividen de nuestros vecinos de sajón origen capitales diferencias que jamás han de permitir que nos unifiquemos en pensamientos y obra" y siguen su argumento visualizando un confrontamiento más violento: "que se sepa que tan enhiestos hemos de erguirnos para rechazar extrañas e ilegítimas influencias, como nos levantaríamos para repeler a un invasor armado". ${ }^{38}$

José Joaquín Terrazas fue, tal vez, el católico más radical y más intransigente de la época. Él veía en los Estados Unidos la causa de todo el mal y todas las desgracias en México ${ }^{39}$. La Doctrina Monroe, escribe Terrazas, es la base ideológica del expansionismo de EEUU y de tal manera hay que frenarlo, más aun por ser una idea no solo "antipolítica", sino antirreligiosa, puesto que intenta obstaculizar toda acción europea en América y también la acción del Vaticano. ${ }^{40}$

Terrazas tampoco deja dudas de sobre quién pesa la responsabilidad por la situación en que está México: "conviene hacer comprender á uno de los partidos en que está dividida la República que debe arrepentirse de las exageradas simpatías que ha manifestado al enemigo de nuestra nacionalidad y nuestra raza".

\footnotetext{
35 "El peligro del Norte", in: El Tiempo, 6 de junio de 1900

${ }^{36}$ Textualmente el artículo dice lo siguiente: "El colosal proyecto del ferrocarril internacional, que unirá estrechamente las repúblicas sud-americanas al coloso del norte; la fundación en Washington de un gran banco, podemos decir pan-americano, con sucursales en todas las Repúblicas del Continente; los grandes syndicatos formados en los más poderosos centros de la Union con el objeto de monopolizar en cuanto sea posible, la explotacion de las industrias de los países hispano-americanos, el ahínco con que se persigue la construcción del canal interoceánico sin intervención de capitales europeos; todos estos proyectos grandiosos, que están actualmente en estudio ó en vías de ejecución, bajo la acción inmediata del gobierno de Washington, ¿qué significan, si no la realización de un vasto plan para asegurar el dominio comercial de los Estados Unidos sobre las demás repúblicas americanas, sobre estas 'insignificantes Repúblicas' como dice el diario neoyorkino antes citado?" ("El peligro del Norte", in: El Tiempo, 6 de junio de 1900)

37 "Dos congresos internacionales", in: El Tiempo, 12 de julio de 1900

${ }^{38}$ La Voz de México, citado por DUMAS, op.cit.: 255

${ }^{39}$ TERRAZAS, José Joaquín: La bandera gudalupano-patriótica, México, Imprenta del Sagrado Corazón de Jesús, 1888: 24

${ }^{40}$ TERRAZAS, José Joaquín: La lucha patriótica entre México, España y los Estados Unidos. En sus relaciones con la cuestión religiosa, México, Imp. Pte. De Sto. Domingo 2, 1897: 8

${ }^{41}$ TERRAZAS, La bandera ...: 29-30
} 
Aquí hay que subrayar que tanto para los liberales, como para los positivistas también los EEUU significaban un peligro y en ese punto hubo poca diferencia entre los tres pensamientos, pero eso ya es tema de otra ponencia. 